\title{
Atividade Física e Saúde - Donde para onde?
}

\author{
Jorge Mota', José Oliveira', José A. Duarte'
}

A importância da atividade física/exercício físico na saúde dos indivíduos e populações é um fato demonstrado em trabalhos recentes e com evidência univer$\mathrm{sal}^{1}$, sugerindo-se mesmo que o exercício físico possa ter uma importância direta na prevenção primária em, pelo menos, 35 estados e/ou condições crónicas ${ }^{2}$. As evidências são, portanto, claras quanto à influência das condições adaptativas da atividade física e do exercício sobre aptidão cardiorrespiratória, da força, da saúde óssea e metabólica em geral ${ }^{3}$ mas, de grande importância, estudos recentes tem indicado pistas e visões novas e adicionais sobre os mecanismos moleculares explicativos dos seus efeitos sobre diferentes estados patológicos ${ }^{4}$.

De forma mais ao menos consistente tem sido ainda evidenciando que a inatividade física e/ou o sedentarismo, normalmente associado ao elevado tempo sentado, é um indicador importante de risco cardiovascular e metabólico especialmente para aqueles sujeitos que não se aproximam do "ser ativo", usando para tanto a referência das recomendações sobre a atividade física ${ }^{5}$. Neste particular, é sugerido uma relação curvilínea entre níveis baixos de atividade física com altos valores de tempo sentado "sitting time" e um aumento significativo da mortalidade 5 .

A questão central, contudo, reside no fato da existência de conhecimento e de consciencialização das populações se traduzir numa maior procura e de realização de atividades físicas e de exercícios físicos mas não encontrar, contudo, uma fórmula de manutenção e de características duradouras e, eventualmente, capazes de um "extensão (scalling-up)" e generalização dessa prática ${ }^{6}$. Sendo, a este propósito, significativo e relevante do ponto de vista social e econômico que os gastos públicos efetivos de despesa com o sector da saúde associado à ausência ou baixos níveis de atividade física, se estimem em valores da ordem dos $\$ 53,8$ biliões de US dólares em todo o mundo ${ }^{7}$. Não é de menosprezar, a este propósito, a questão basilar na qual a ausência de recursos financeiros e /ou nível socioeconômicos baixos (educacional, por exemplo) se traduzem em indicadores importantes de risco, não apenas comportamental, mas também de morbilidade e mortalidade ${ }^{8}$.

Tendo em consideração as questões centrais aludidas previamente, parece-nos que os pontos basilares de desenvolvimento da investigação na área da atividade física, exercício físico e saúde estão centradas num conjunto de evidências e de investigação que nos permita uma melhor compreensão e/ou definição: (1) Dos mecanismos celulares e moleculares que exprimem, cronicamente, a adaptação /ou estimulação do organismo em relação ao exercício físico, permitindo a identificação de novas terapias e eventuais alvos para o desenvolvimento dessas terapias no tratamento de diferente patologias ${ }^{6}$; (2) O problema efetivo da intensidade do exercício físico/atividade física enquanto referência, não apenas acadêmica mas 
também populacional, bem como das suas repercussões efetivas na adaptação fisiológica dos indivíduos. Há, hoje em dia, evidência importante que as atividades físicas de menor gasto energético, como atividades "ligeiras", são importantes e podem ter um efeito protetivo positivo ${ }^{9}$. Contudo, não é desprezível a qualidade da intensidade no âmbito do dispêndio energético e das repercussões metabólicas, verificando-se uma importância específica das atividades mais vigorosas na proteção cardio-metabólica ${ }^{10}$; (3) De todo o modo, as práticas de exercício físico e/ou de atividade física carecem de um envolvimento comportamental que se materializa num conjunto de determinantes (fatores intrapessoais como o prazer na prática; fatores e interpessoais como o suporte social; fatores culturais como a aculturação; fatores ambientais, como a existência de parques e ou infra estruturas para a prática; fatores /decisões políticas como a existência de aulas de educação física obrigatórias), estruturantes no âmbito motivacional e psicológico, para a prática ${ }^{11}$. Como nos é bem evidenciado é encorajador o fato de que mesmo que seja incompatível para as pessoas, por múltiplas razões, o evitar de longos períodos de inatividade (sitting) durante o dia, esta situação pode ser "compensada" por níveis adequados de prática de atividades físicas/exercícios em outros momentos do dia ${ }^{5}$. Este é um resultado importante do ponto de vista do esclarecimento dos impedimentos efetivos para as pessoas no que respeita a uma prática mais consistente que nos leva para o campo da gestão do tempo pessoal e da importância do controlo comportamental ${ }^{11}$ e do estudo do tempo compósito tendo em conta a finitude temporal em que se concretiza a vida quotidiana ${ }^{12}$. (4) A necessidade de clarificar e definir de forma mais robusta a dose efetiva bem como o tipo de exercício físico que são requeridos para produzir benefícios para a saúde em função dos diferentes "outcomes" que lhe são associados ${ }^{13}$; Nesse contexto, (5) as recomendações associadas aos benefícios devem ser fortalecidas por mais estudos longitudinais que possam considerar a diversidade das populações e contexto em estudo no sentido de poderem dar um contributo importante às questões mencionadas anteriormente, nomeadamente na dose-resposta; na análise e desenvolvimento dos projetos e da sua avaliação bem como no sustentar das políticas de monitorização e de vigilância ${ }^{11}$. Não é de desprezar o interesse crescente, sustentados nem novos "statments", traduzindo-se na necessidade que toda a produção científica bem como a consciencialização pública sobre os benefícios do "ser ativo" possam ser materializados nas recomendações sobre a atividade física incorporando medidas efetivas na redução do tempo sedentário ${ }^{1}$; Finalmente, (6) a incorporação de qualitativamente melhores instrumentos de avaliação objetiva da atividade física no sentido de uma melhoria dos comportamentos bem como da sua manutenção a longo prazo são um desafio promissor. De particular relevância o desenvolvimento de pequenos aparelhos que permitam a captação da informação "in loco" e contextualizada dos comportamentos sejam eles "ativos" ou "sedentários", no sentido de elucidar a complexa interação comportamental dos sujeitos.

\section{Referências}

1. Andersen LB, Mota J, Di Pietro L. Update on the global pandemic of physical inactivity. Lancet (London, England). 2016;388:1255-6.

2. Booth FW, Roberts CK, Laye MJ. Lack of exercise is a major cause of chronic diseases. Compr Physiol. 2012;2:1143-211.

3. Fiuza-Luces C, Garatachea N, Berger NA, Lucia A. Exercise is the real polypill. Physiology (Bethesda, Md). 2013;28:330-58. 
4. Febbraio MA. Exercise metabolism in 2016: Health benefits of exercise - more than meets the eye! Nat Rev Endocrinol. 2017;13:72-4.

5. Ekelund U, Steene-Johannessen J, Brown WJ, Fagerland MW, Owen N, Powell KE, et al. Does physical activity attenuate, or even eliminate, the detrimental association of sitting time with mortality? A harmonised meta-analysis of data from more than 1 million men and women. Lancet (London, England). 2016;388:1302-10.

6. Reis RS, Salvo D, Ogilvie D, Lambert EV, Goenka S, Brownson RC. Scaling up physical activity interventions worldwide: stepping up to larger and smarter approaches to get people moving. Lancet (London, England). 2016;388:1337-48.

7. Ding D, Lawson KD, Kolbe-Alexander TL, Finkelstein EA, Katzmarzyk PT, van Mechelen $\mathrm{W}$, et al. The economic burden of physical inactivity: a global analysis of major noncommunicable diseases. Lancet (London, England). 2016;388:1311-24.

8. Stringhini S, Carmeli C, Jokela M, Avendano M, Muennig P, Guida F, et al. Socioeconomic status and the $25 \times 25$ risk factors as determinants of premature mortality: a multicohort study and meta-analysis of 1.7 million men and women. Lancet. 2017, 5(3):e230-e231 (London, England). 2017.

9. Engelen L, Gale J, Chau JY, Hardy LL, Mackey M, Johnson N, et al. Who is at risk of chronic disease? Associations between risk profiles of physical activity, sitting and cardiometabolic disease in Australian adults. Aust N Z J Public Health. 2017; 41(2):178-183.

10. Janssen I, Ross R. Vigorous intensity physical activity is related to the metabolic syndrome independent of the physical activity dose. Int J Epidemiol. 2012;41:1132-40.

11. Sallis JF, Bull F, Guthold R, Heath GW, Inoue S, Kelly P, et al. Progress in physical activity over the Olympic quadrennium. Lancet (London, England). 2016;388:1325-36.

12. Chastin SF, Palarea-Albaladejo J, Dontje ML, Skelton DA. Combined Effects of Time Spent in Physical Activity, Sedentary Behaviors and Sleep on Obesity and CardioMetabolic Health Markers: A Novel Compositional Data Analysis Approach. PloS one. 2015;10:e0139984.

13. Silva KS, Garcia LM, Rabacow FM, de Rezende LF, de Sa TH. Physical activity as part of daily living: Moving beyond quantitative recommendations. Prev Med. 2017;96:160-2.

Endereço para

correspondência

Jorge Mota

Rua Dr Placido Costa, 91

4200-450. Porto.

Portugal.

jmota@fade.Up.Pt

Fone: +351-220425245 